\title{
Quantization of scalar perturbations in brane-world inflation
}

\author{
Hiroyuki Yoshiguchi ${ }^{1}$ and Kazuya Koyama ${ }^{1,2}$ \\ 1 Department of Physics, University of Tokyo 7-3-1 Hongo, Bunkyo, Tokyo 113-0033, Japan and \\ ${ }^{2}$ Institute of Cosmology and Gravitation, Portsmouth University, Portsmouth, PO1 2EG, UK
}

\begin{abstract}
We consider a quantization of scalar perturbations about a de Sitter brane in a 5-dimensional anti-de Sitter (AdS) bulk spacetime. We first derive the second order action for a master variable $\Omega$ for 5-dimensional gravitational perturbations. For a vacuum brane, there is a continuum of normalizable Kaluza-Klein (KK) modes with $m>3 H / 2$. There is also a light radion mode with $m=\sqrt{2} H$ which satisfies the junction conditions for two branes, but is non-normalizable for a single brane model. We perform the quantization of these bulk perturbations and calculate the effective energy density of the projected Weyl tensor on the barne. If there is a test scalar field perturbation on the brane, the $\mathrm{m}^{2}=2 \mathrm{H}^{2}$ mode together with the zero-mode and an infinite ladder of discrete tachyonic modes become normalizable in a single brane model. This infinite ladder of discrete modes as well as the continuum of KK modes with $m>3 H / 2$ introduce corrections to the scalar field perturbations at first-order in a slow-roll expansion. We derive the second order action for the Mukhanov-Sasaki variable coupled to the bulk perturbations which is needed to perform the quantization and determine the amplitude of scalar perturbations generated during inflation on the brane.
\end{abstract}

PACS numbers: $98.80 . \mathrm{Cq}, 04.50 .+\mathrm{h}$

\section{INTRODUCTION}

In recent years, there has been a great deal of interests in the brane-world picture where ordinary matter fields are confined to a lower-dimensional hypersurface (brane) embedded in a higher-dimensional bulk spacetime (see [1, 2, 3, 4] for reviews). In particular, models proposed by Randall and Sundrum are very interesting [5, 6]. In their second model (RS model), they showed that the conventional 4-dimensional gravity can be recovered at low energies on a Minkowski brane with positive tension in 5-dimensional anti-de Sitter (AdS) spacetime [6]. An interesting point is that the 4-dimensional gravity can be recovered despite the infinite size of the extra-dimension.

At low energies, effects of the extra-dimension must be small from the observational point of view, but at high energies the effects could be dominant. The effects of the extra-dimension could affect generations of primordial fluctuations in the period of inflation in the early universe. Such imprints left on the spectrum of primordial fluctuations will be constrained by high precision observations of the Cosmic Microwave Background (CMB).

In general, it is difficult to find analytic solutions for cosmological perturbations that properly satisfy the junction conditions on the brane 7, 8. However, this can be done in the special case of a de Sitter brane in a 5-dimensional AdS bulk spacetime [9, 10]. This fact is useful since it provides a zero-th order approximation for slow-roll inflation on the brane. The behavior of vector [1] and tensor [12, 13, 14, 15] perturbations have been discussed already .

The amplitude of scalar perturbations excited by inflaton fluctuations on the brane is also computed in the extreme slow-roll limit where the coupling between inflaton field fluctuations and bulk perturbations can be neglected [16, 17]. However, to go beyond the zero-th order slow-roll approximation, we have to solve the bulk metric perturbations. This issue is recently discussed by Koyama et al. [18] within a classical theory, using a master variable $\Omega$ for gravitational perturbations in a 5-dimensional AdS spacetime [19, 20]. They showed that the bulk perturbations introduce corrections to the scalar field fluctuations at first order computed in a slow-roll expansion. Thus, to quantitatively calculate the amplitude of scalar perturbations at this order, we need to discuss a quantum theory of the bulk gravitational field as well as the inflaton field on the brane.

In this paper, we study the quantization of bulk scalar metric perturbations about a de Sitter brane in the RS model. We review the background spacetime in SecIII In SecIIII we then introduce the master variable $\Omega$ for gravitational perturbations in 5-dimensional AdS spacetime [19, 20] and derive its second order action (34). Then we first consider quantum scalar perturbations in the absence of matter fields on the brane in Sec IV In this case, there is a continuum of normalizable KK modes with $m>3 H / 2$. These bulk perturbations are felt on the brane through the projection of the perturbed 5-dimensional Weyl tensor $\delta E_{\mu \nu}$. We calculate the vacuum expectation value of its effective energy density by using the second order action for $\Omega$. A light radion mode with $m=\sqrt{2} H$ also satisfies the junction condition on the branes, and is normalizable for a two branes model [18, 21, 22]. The effective energy density of $\delta E_{\mu \nu}$ due to this radion mode is also calculated. Next we consider bulk scalar perturbations excited by scalar field fluctuations on the brane in Sec $\nabla$ We first discuss the effect of the metric backreaction on the scalar field fluctuations and review the solution for the bulk perturbations derived in Ref. [18] in Sec. VA We show that the evolution equation for the Mukhanov-Sasaki variable has a correction term that comes from the bulk perturbations. 
To quantize the Mukhanov-Sasaki variable, we need its action coupled to the bulk gravitational field. We derive this action (99) in Sec $\nabla \mathrm{B}$. We summarize the main results and discuss them in Sec. VI

\section{COSMOLOGICAL BACKGROUND}

The action describing the RS brane world is given by

$$
S=\int d^{5} x \sqrt{-{ }^{(5)} g}\left[\frac{1}{2 \kappa^{2}}\left({ }^{(5)} R+12 \mu^{2}\right)\right]+\int d^{4} x \sqrt{-g}\left[-\lambda+\mathcal{L}_{\text {matter }}+\frac{1}{\kappa^{2}} K\right],
$$

where $\kappa^{2}$ is 5 -dimensional gravitational constant, and $\mu$ is the curvature scale of the AdS spacetime. The brane has tension $\lambda$ which is set to be $6 \mu / \kappa^{2}$ in the RS model. The induced metric on the brane is denoted as $g$, and given by $g_{A B}={ }^{(5)} g_{A B}-n_{A} n_{B}$, where $n^{A}$ is the unit vector normal to the brane. Matter field is confined on the 3 -brane and

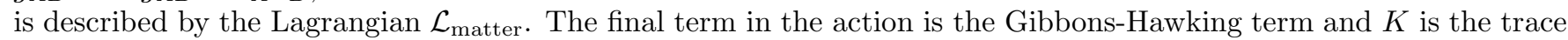
of the extrinsic curvature of the brane. We assume the $Z_{2}$ symmetry across the brane.

The 5-dimensional Einstein equations are obtained by minimizing the action with respect to variations of the bulk metric:

$$
{ }^{(5)} G_{A B}+{ }^{(5)} g_{A B} \Lambda_{5}=0
$$

The 4-dimensional matter fields determine the brane trajectory in the AdS bulk through the junction condition by producing the jump in the extrinsic curvature at the brane. The surface energy-momentum on the brane can be split into two parts, $T_{\mu \nu}-\lambda g_{\mu \nu}$, where $T_{\mu \nu}$ is taken to be the matter energy-momentum tensor and $\lambda$ a constant brane tension. The junction condition is then [23, 24]

$$
\left[K_{\nu}^{\mu}\right] \equiv K_{\nu}^{\mu+}-K_{\nu}^{\mu-}=-\kappa_{5}^{2}\left(T_{\nu}^{\mu}-\frac{1}{3} g_{\nu}^{\mu}(T-\lambda)\right)
$$

where the extrinsic curvature of the brane is denoted by $K_{\mu \nu}=g_{\mu}{ }^{C} g_{\nu}{ }^{D}\left({ }^{(5)} \nabla_{C} n_{D}\right)$, where ${ }^{(5)} \nabla_{C}$ is the $5 \mathrm{D}$ covariant derivative. The induced Einstein equations on the brane are then given by [24]

$$
G_{\mu \nu}=\kappa_{4}^{2} T_{\mu \nu}+\kappa^{4} \Pi_{\mu \nu}-E_{\mu \nu}
$$

where $\kappa_{4}^{2}=\mu \kappa^{2}$. This equation include the projected five-dimensional Wey tensor $E_{\mu \nu}$ as well as the high energy correction term $\Pi_{\mu \nu}$ which is quadratic in the energy-momentum tensor $T_{\mu \nu}$.

In this paper, we consider inhomogeneous bulk metric perturbations in the special case of a de Sitter brane in the AdS bulk. This is a good approximation to a brane-world inflation model with a slow-rolling scalar field on the brane. The metric of the unperturbed 5D spacetime in terms of the conformal bulk-coordinate is given by

$$
d s^{2}=e^{2 W(z)}\left[d z^{2}-d t^{2}+e^{2 \alpha(t)} \delta_{i j} d x^{i} d x^{j}\right]
$$

where

$$
e^{\alpha(t)}=\exp (H t), e^{W(z)}=\frac{H}{\mu \sinh H z}
$$

and $H(=\dot{\alpha}(t))$ is a constant Hubble expansion rate on the brane. The Cauchy horizon is located at $|z|=\infty$, and the brane is located at $z=z_{0}$ given by

$$
z_{0}=\frac{1}{H} \sinh ^{-1} \frac{H}{\mu}
$$

The background metric satisfies

$$
\begin{aligned}
& H^{2}+W^{\prime \prime}-W^{2}=0 \\
& W^{\prime \prime}=\mu^{2} e^{2 W}
\end{aligned}
$$




\section{MASTER VARIABLE AND SECOND ORDER ACTION}

\section{A. Master variable}

Here we introduce the master variable for gravitational perturbations in the 5-dimensional AdS spacetime firstly found by Mukohyama [19, 20]. The perturbed metric is given by [25]

$$
\begin{aligned}
d s^{2}= & e^{2 W(z)}\left[\left(1+2 A_{y y}\right) d z^{2}+2 A_{y} d t d z-(1+2 A) d t^{2}\right. \\
& \left.+e^{2 \alpha(t)}\left((1+2 \mathcal{R}) \delta_{i j} d x^{i} d x^{j}+2 E_{, i j} d x^{i} d x^{j}+2 B_{, i} d x^{i} d t+2 C_{, i} d x^{i} d z\right)\right] .
\end{aligned}
$$

Under a scalar type gauge transformation,

$$
\begin{aligned}
& t \rightarrow \bar{t}=t+\xi^{t} Y, \\
& z \rightarrow \bar{z}=z+\xi^{z} Y, \\
& x^{i} \rightarrow \bar{x}^{i}=x^{i}+\xi^{S} Y^{i},
\end{aligned}
$$

the metric variables transform as

$$
\begin{aligned}
A_{y y} & \rightarrow \bar{A}_{y y}=A_{y y}-\xi^{z \prime}-W^{\prime} \xi^{z} \\
A_{y} & \rightarrow \bar{A}_{y}=A_{y}+\xi^{t \prime}-\dot{\xi}^{z} \\
C & \rightarrow \bar{C}=C-e^{-2 \alpha} \xi^{z}-\xi^{S \prime} \\
A & \rightarrow \bar{A}=A-W^{\prime} \xi^{z}-\dot{\xi}^{t} \\
B & \rightarrow \bar{B}=B+e^{-2 \alpha} \xi^{t}-\dot{\xi}^{S} \\
E & \rightarrow \bar{E}=E-\xi^{S} \\
\mathcal{R} & \rightarrow \overline{\mathcal{R}}=\mathcal{R}-W^{\prime} \xi^{z}-\dot{\alpha} \xi^{t}
\end{aligned}
$$

where dot and prime denote the derivative with respect to $t$ and $z$, respectively. In order to eliminate the gauge dependence on choice of 3-space coordinates we introduce the spatially gauge-invariant combinations

$$
\begin{aligned}
\sigma_{t} & \equiv-B+\dot{E} \\
\sigma_{z} & \equiv-C+E^{\prime}
\end{aligned}
$$

which are subject only to temporal and bulk gauge transformations.

The bulk and temporal gauges are fully determined by setting $\sigma_{t}=\sigma_{z}=0$, which has been termed the 5Dlongitudinal gauge [25, 26]. We can define the remaining metric perturbations in the 5D-longitudinal gauge as

$$
\begin{aligned}
\Phi & =A-\left(e^{2 \alpha} \sigma_{t}\right)^{\cdot}+W^{\prime} e^{2 \alpha} \sigma_{z} \\
\Psi & =\mathcal{R}-\dot{\alpha} e^{2 \alpha} \sigma_{t}+W^{\prime} e^{2 \alpha} \sigma_{z}, \\
S & =A_{y}+e^{2 \alpha} \sigma_{t}^{\prime}+\left(e^{2 \alpha} \sigma_{z}\right)^{\cdot} \\
N & =A_{y y}+e^{2 \alpha}\left(\sigma_{z}^{\prime}+W^{\prime} \sigma_{z}\right) .
\end{aligned}
$$

These are equivalent to the gauge-invariant bulk perturbations originally introduced in covariant form by Mukohyama [19, 20] and in a coordinate-based approach by van den Bruck et al [26]. These metric variables satisfy the three constraint equations,

$$
\begin{aligned}
& N+\Phi+\Psi=0 \\
& -\Phi^{\prime}-2 \Psi^{\prime}+3 W^{\prime} N-\frac{1}{2}(\dot{S}+H S)=0 \\
& -\dot{N}+H N-2 \dot{\Psi}+2 H \Phi+\frac{1}{2}\left(S^{\prime}+3 W^{\prime} S\right)=0 .
\end{aligned}
$$

In [19] (see also Ref. 20]), it was shown that the perturbed 5D Einstein equations, in the absence of bulk matter perturbations are solved in an AdS background using a "master variable", $\Omega$. In the special case of a de Sitter brane 
in the AdS bulk, the metric variables are written by the master variable $\Omega$ as

$$
\begin{aligned}
& \Phi=-\frac{e^{-\alpha-3 W}}{6}\left(2 \Omega^{\prime \prime}-3 W^{\prime} \Omega^{\prime}+\ddot{\Omega}-\mu^{2} e^{2 W} \Omega\right) \\
& S=e^{-\alpha-3 W}\left(\dot{\Omega}^{\prime}-W^{\prime} \dot{\Omega}\right) \\
& N=\frac{e^{-\alpha-3 W}}{6}\left(\Omega^{\prime \prime}-3 W^{\prime} \Omega^{\prime}+2 \ddot{\Omega}+\mu^{2} e^{2 W} \Omega\right) \\
& \Psi=\frac{e^{-\alpha-3 W}}{6}\left(\Omega^{\prime \prime}-\ddot{\Omega}-2 \mu^{2} e^{2 W} \Omega\right)
\end{aligned}
$$

These expressions satisfy the constraint equations Eq. 17 19).

Now, we derive the equation of motion for the master variable $\Omega$. By substituting the expression Eq. (20) 23) into the dynamical parts of the perturbed Einstein equations, we can show that they are equivalent to [19]

$$
\begin{aligned}
\Delta_{(S)}^{\prime \prime}-W^{\prime} \Delta_{(S)}^{\prime}-\mu^{2} e^{2 W} \Delta_{(S)} & =0 \\
\ddot{\Delta}_{(S)}-W^{\prime} \Delta_{(S)}^{\prime}+\mu^{2} e^{2 W} \Delta_{(S)} & =0 \\
\dot{\Delta}_{(S)}^{\prime}-W^{\prime} \dot{\Delta}_{(S)} & =0
\end{aligned}
$$

where

$$
\Delta_{(S)}=e^{2 \alpha}\left[\ddot{\Omega}-3 H \dot{\Omega}-\left(\Omega^{\prime \prime}-3 W^{\prime} \Omega^{\prime}\right)-\mu^{2} e^{2 W} \Omega-e^{-2 \alpha} \Delta \Omega\right] .
$$

Here $\Delta$ is the 3D spatial Laplacian $\Delta \Omega=\delta^{i j} \Omega_{, i j}$. Then, we can obtain the following equation from Eq.(24):

$$
\ddot{\tilde{\Omega}}-3 H \dot{\tilde{\Omega}}-\left(\tilde{\Omega}^{\prime \prime}-3 W^{\prime} \tilde{\Omega}^{\prime}\right)-e^{-2 \alpha} \Delta \tilde{\Omega}-\mu^{2} e^{2 W} \tilde{\Omega}=0
$$

where

$$
\Delta \tilde{\Omega}=\Delta \Omega+\Delta(S)
$$

Here we note that there is a symmetry between $\Omega$ and $\tilde{\Omega}$. It is possible to show that a replacement of $\Omega$ with $\tilde{\Omega}$ in Eq. 20,23) does not alter the metric variables if the perturbed Einstein equations Eq.(24) are satisfied. Then we can set $\Delta_{(S)}=0$ if $\Delta \Omega \neq 0$ as is shown in Ref. [19]. This is equivalent to say that the solutions of the metric variables are given by Eq. (20,23) where $\Omega$ is a solution of the master equation:

$$
\ddot{\Omega}-3 H \dot{\Omega}-\left(\Omega^{\prime \prime}-3 W^{\prime} \Omega^{\prime}\right)-e^{-2 \alpha} \Delta \Omega-\mu^{2} e^{2 W} \Omega=0 .
$$

We also see that $\tilde{\Omega}$ coincides with $\Omega$ if we use Eq.(28).

\section{B. Second order action for master variable}

In this subsection, we derive the second order action for $\Omega$ including the surface terms on the brane. By perturbing the gravitational part (except for $\mathcal{L}_{\text {matter}}$ ) of the action Eq.(1) up to second order, we get

$$
\begin{aligned}
\delta_{2} S= & \int d^{5} x \frac{e^{3 \alpha+3 W}}{\kappa^{2}}\left[3\left(H^{2}+W^{\prime \prime}+3 W^{\prime 2}\right) A_{y y}^{2}-6 H^{2} A^{2}-6 H^{2} A_{y y} A-3 H^{2} A_{y}^{2}\right. \\
& -2 e^{-2 \alpha}\left(A \Delta A_{y y}+2 \mathcal{R} \Delta A_{y y}+\mathcal{R} \Delta \mathcal{R}+2 A \Delta \mathcal{R}\right) \\
& -6 W^{\prime} A^{\prime} A_{y y}+6 H \dot{A}_{y y} A+6 W^{\prime} \dot{A}_{y y} A_{y}+6 H A^{\prime} A_{y}-\frac{1}{2} e^{-2 \alpha} A_{y} \Delta A_{y} \\
& -2 A_{y}\left(3 \dot{\mathcal{R}}^{\prime}+\Delta \dot{E}^{\prime}-\Delta \dot{C}\right)-\frac{1}{2} e^{2 \alpha}\left(B^{\prime}-\dot{C}-2 e^{-2 \alpha} A_{y}\right) \Delta\left(B^{\prime}-\dot{C}\right) \\
& -6 \mathcal{R}^{\prime 2}+6 \dot{\mathcal{R}}^{2}-2(3 \dot{\mathcal{R}}+\Delta \dot{E}-\Delta B)\left(\dot{A}_{y y}-H A_{y y}+2 \dot{\mathcal{R}}-2 H A\right) \\
& \left.+2\left(3 \mathcal{R}^{\prime}+\Delta E^{\prime}-\Delta C\right)\left(A^{\prime}+2 \mathcal{R}^{\prime}-3 W^{\prime} A_{y y}-H A_{y}\right)\right]
\end{aligned}
$$


Here any gauge conditions are not imposed. The surface term on the brane cancels out. This action can be simplified by imposing the 5D-longitudinal gauge and using the constraint equations Eq.(17)-(19) as

$$
\begin{aligned}
\delta_{2} S= & \int d^{5} x \frac{e^{3 \alpha+3 W}}{\kappa^{2}}\left[3\left(H^{2}+W^{\prime \prime}+3 W^{\prime 2}\right) N^{2}-6 H^{2} \Phi^{2}-6 H^{2} N \Phi-3 H^{2} S^{2}\right. \\
& -2 e^{-2 \alpha}(\Phi \Delta N+2 \Psi \Delta N+\Psi \Delta \Psi+2 \Phi \Delta \Psi) \\
& \left.-6 W^{\prime} \Phi^{\prime} N+6 H \dot{N} \Phi+6 W^{\prime} \dot{N} S+6 H \Phi^{\prime} S-\frac{1}{2} e^{-2 \alpha} S \Delta S-6 \Psi^{\prime 2}+6 \dot{\Psi}^{2}\right] \\
& +\int d^{4} x \frac{e^{3 \alpha}}{\kappa^{2}}[3 S \dot{\Psi}] .
\end{aligned}
$$

To obtain the second order action for $\Omega$, we first substitute the expressions of the metric variables in terms of $\Omega$, Eq.(20)-(23). After integrating by parts, we get the following action

$$
\begin{aligned}
S=\int & d^{5} x \frac{e^{\alpha-3 W}}{6 \kappa^{2}}\left[\dddot{\Omega}^{2}-\Omega^{\prime \prime \prime 2}+3 \dot{\Omega}^{\prime \prime 2}-3 \ddot{\Omega}^{\prime 2}+e^{-2 \alpha}\left(\ddot{\Omega} \Delta \ddot{\Omega}+\Omega^{\prime \prime} \Delta \Omega^{\prime \prime}-2 \dot{\Omega}^{\prime} \Delta \dot{\Omega}^{\prime}\right)\right. \\
& +\left(14 H^{2}+3 W^{\prime \prime}\right) \ddot{\Omega}^{2}-2\left(H^{2}+3 W^{\prime \prime}\right) \Omega^{\prime \prime 2}+3\left(-4 H^{2}+W^{\prime \prime}\right) \dot{\Omega}^{\prime 2} \\
& +3\left(7 W^{\prime \prime 2}-14 W^{\prime 2} W^{\prime \prime}+8 W^{\prime 4}\right) \dot{\Omega}^{2}+\left(5 W^{\prime \prime 2}-22 W^{\prime 2} W^{\prime \prime}+8 W^{\prime 4}\right) \Omega^{2}+9 W^{\prime \prime} \Omega^{2} \\
& \left.+2\left(3 H^{2}+W^{\prime \prime}\right) e^{-2 \alpha} \dot{\Omega} \Delta \dot{\Omega}+\left(-2 H^{2}+W^{\prime \prime}\right) e^{-2 \alpha} \Omega^{\prime} \Delta \Omega^{\prime}+W^{\prime \prime 2} e^{-2 \alpha} \Omega \Delta \Omega\right] \\
- & \int d^{4} x \frac{e^{\alpha}}{6 \kappa^{2}}\left[5 \ddot{\Omega}^{\prime} \Omega^{\prime \prime}-\ddot{\Omega}^{\prime} \ddot{\Omega}+4 W^{\prime} \Omega^{\prime \prime 2}+4 W^{\prime} \ddot{\Omega}^{2}-5 W^{\prime} \Omega^{\prime \prime} \ddot{\Omega}+\frac{3}{2} W^{\prime} \dot{\Omega}^{2}-H \dot{\Omega}^{\prime} \ddot{\Omega}-6 H \dot{\Omega}^{\prime} \Omega^{\prime \prime}\right. \\
& +\left(4 W^{\prime \prime}-6 W^{\prime 2}\right) \Omega^{\prime} \Omega^{\prime \prime}+6 H W^{\prime} \dot{\Omega} \Omega^{\prime \prime}-\left(11 H^{2}+5 W^{\prime \prime}\right) \dot{\Omega}^{\prime} \dot{\Omega}-2 W^{\prime} W^{\prime \prime} \Omega \Omega^{\prime \prime} \\
& +W^{\prime}\left(-\frac{3}{2} H^{2}+4 W^{\prime \prime}\right) \Omega^{\prime 2}+W^{\prime}\left(12 H^{2}-\frac{11}{2} W^{\prime \prime}\right) \dot{\Omega}^{2}-H\left(17 H^{2}+22 W^{\prime \prime}\right) \dot{\Omega} \Omega^{\prime} \\
& +W^{\prime \prime}\left(9 H^{2}+8 W^{\prime \prime}\right) \Omega \Omega^{\prime}+W^{\prime} W^{\prime \prime}\left(\frac{5}{2} H^{2}-5 W^{\prime \prime}\right) \\
& \left.+e^{-2 \alpha}\left(-\dot{\Omega}^{\prime} \Delta \dot{\Omega}+H \Omega^{\prime} \Delta \dot{\Omega}-W^{\prime \prime} \Omega^{\prime} \Delta \Omega-\frac{1}{2} W^{\prime} W^{\prime \prime} \Omega \Delta \Omega-\frac{3}{2} W^{\prime} \Omega^{\prime} \Delta \Omega^{\prime}+3 W^{\prime} \dot{\Omega} \Delta \dot{\Omega}\right)\right]
\end{aligned}
$$

Although this action is extremely long, we can summarize the result in the following form:

$$
\begin{aligned}
S & =\int d^{5} x \frac{e^{-3 \alpha-3 W}}{6 \kappa^{2}}\left[(\Delta \dot{\tilde{\Omega}})^{2}-\left(\Delta \tilde{\Omega}^{\prime}\right)^{2}+e^{-2 \alpha} \Delta \tilde{\Omega} \Delta^{2} \tilde{\Omega}+\mu^{2} e^{2 W}(\Delta \tilde{\Omega})^{2}\right] \\
& +\int d^{4} x \frac{e^{\alpha}}{6 \kappa^{2}}\left[\frac{9}{2} W^{\prime} \dot{\mathcal{F}}^{2}-\frac{3}{2} W^{\prime} e^{-2 \alpha} \mathcal{F} \Delta \mathcal{F}-W^{\prime} e^{-4 \alpha}(\Delta \tilde{\Omega})^{2}-3 \ddot{\mathcal{F}} e^{-2 \alpha} \Delta(\Omega-\tilde{\Omega})\right],
\end{aligned}
$$

where

$$
\mathcal{F}=\Omega^{\prime}-W^{\prime} \Omega
$$

and $\tilde{\Omega}$ is defined in Eq. (27). This action contains higher derivative terms with respect to $t$. In order to perform a quantization of $\Omega$, we need an action that contains up to second derivatives. This can be achieved using the symmetry between $\tilde{\Omega}$ and $\Omega$. It is possible to replace $\tilde{\Omega}$ with $\Omega$ or equivalently we can set $\Delta_{(S)}=0$ without losing the physical degrees of freedom [19]. Then we can derive the second order action for $\Omega$ that contains up to second derivatives with respect to $t$ :

$$
\begin{aligned}
S & =\int d^{5} x \frac{e^{-3 \alpha-3 W}}{6 \kappa^{2}}\left[(\Delta \dot{\Omega})^{2}-\left(\Delta \Omega^{\prime}\right)^{2}+e^{-2 \alpha} \Delta \Omega \Delta^{2} \Omega+\mu^{2} e^{2 W}(\Delta \Omega)^{2}\right] \\
& +\int d^{4} x \frac{e^{\alpha}}{6 \kappa^{2}}\left[\frac{9}{2} W^{\prime} \dot{\mathcal{F}}^{2}-\frac{3}{2} W^{\prime} e^{-2 \alpha} \mathcal{F} \Delta \mathcal{F}-W^{\prime} e^{-4 \alpha}(\Delta \Omega)^{2}\right] .
\end{aligned}
$$

Defining

$$
\omega=\frac{e^{-3 \alpha-3 W}}{\sqrt{3 \kappa^{2}}} \Delta \Omega
$$


the second order action for $\Omega$ in the bulk can be rewritten into the form of a 5-dimensional scalar field:

$$
S=\frac{1}{2} \int d^{5} x e^{3 \alpha+3 W}\left[\dot{\omega}^{2}-\omega^{2}+e^{-2 \alpha} \omega \Delta \omega+4 W^{\prime \prime} \omega^{2}\right] .
$$

This form of the action is convenient when we fix the normalization of $\Omega$.

\section{General solution for master variable}

In this subsection, we derive the general solution for $\Omega$. Solutions of the wave equation Eq. (28) can be separated into eigenmodes of the time-dependent equation on the brane and bulk mode equation:

$$
\Omega(t, y ; \vec{x})=\int d^{3} \vec{k} d m v_{m}(t) u_{m}(y) e^{i \vec{k} \cdot \vec{x}}
$$

where

$$
\begin{aligned}
\ddot{v}_{m}-3 H \dot{v}_{m}+\left[m^{2}+k^{2} e^{-2 \alpha}\right] v_{m} & =0, \\
u_{m}^{\prime \prime}-3 W^{\prime} u_{m}^{\prime}+\left[m^{2}+\mu^{2} e^{2 W}\right] u_{m} & =0 .
\end{aligned}
$$

The general solution for $v_{m}$ is given by

$$
v_{m}(\eta ; \vec{k})=(-k \eta)^{-3 / 2} B_{\nu}(-k \eta), \quad \nu^{2}=\frac{9}{4}-\frac{m^{2}}{H^{2}},
$$

where $B_{\nu}$ is a linear combination of Bessel functions of order $\nu$.

Defining $\Psi_{m} \equiv e^{-3 W / 2} u_{m}$, it is possible to rewrite the off-brane equation (39) in Schrödinger-like form:

$$
\frac{d^{2} \Psi_{m}}{d z^{2}}-V \Psi_{m}=-m^{2} \Psi_{m}
$$

where

$$
\begin{aligned}
V(z) & =-\frac{1}{4} \mu^{2} e^{2 W(z)}+\frac{9}{4} H^{2}, \\
& =-\frac{H^{2}}{4 \sinh ^{2}(H z)}+\frac{9}{4} H^{2} .
\end{aligned}
$$

For $z \rightarrow \infty$ we have $V \rightarrow 9 H^{2} / 4$ and we have a continuum of massive modes for $m^{2}>9 H^{2} / 4$ which become oscillating plane waves as $z \rightarrow \infty$. The general solution of the mode function in the $z$-direction is

$$
u_{m}(z)=(\sinh H z)^{-1} W_{\nu-1 / 2}(\cosh H z),
$$

where $W_{\alpha}$ is a linear combination of Legendre functions of order $\alpha$.

\section{QUANTUM GRAVITON WITH A VACUUM DE SITTER BRANE}

We first consider a quantization of scalar perturbations in the absence of matter perturbations. If we take the variation of the action Eq.(34) with respect to $\Omega$, we get the equation of motion for $\Omega$ in the bulk Eq.(28) and the junction condition on the brane

$$
\mathcal{F}=0 \text {. }
$$

This is consistent with the condition obtained by minimizing the action with respect to $\mathcal{F}$.

The effect of bulk perturbations is felt through the projected Weyl tensor $E_{\mu \nu}$ in Eq. (4). In the background spacetime $E_{\mu \nu}=0$ and the energy density of perturbed projected Weyl tensor $\delta E_{\mu \nu}$ is given in terms of the master variable by $[27]$

$$
\kappa_{4}^{2} \delta \rho_{E}=\frac{k^{4} e^{-5 \alpha}}{3} \Omega
$$


In order to estimate the effect of Weyl tensor, we will compare the Weyl energy density perturbation with the background energy density given by

$$
\rho_{\Lambda}=\frac{3 H^{2}}{\kappa_{4, \mathrm{eff}}^{2}}, \quad \kappa_{4, \mathrm{eff}}^{2}=\kappa_{4}^{2}\left[1+\left(\frac{H}{\mu}\right)^{2}\right]^{1 / 2} .
$$

We define the power spectrum $P_{E}(k)$ of $\delta \rho_{E}$ normalized by $\rho_{\Lambda}$ :

$$
\frac{<\delta \rho_{E}^{2}>}{\rho_{\Lambda}^{2}}=\int \frac{d k}{k} P_{E}(k)
$$

\section{A. Quantization of heavy modes}

Here we consider the quantization of the heavy modes with $m>\frac{3}{2} H$. The junction condition for $\Omega$ on the brane is given by Eq.(44). From this condition, the solution of the bulk mode function is written as

$$
u_{m}(z)=C(m)(\sinh H z)^{-1}\left(P_{i \gamma-1 / 2}(\cosh H z)+\beta(m) Q_{i \gamma-1 / 2}(\cosh H z)\right)
$$

where

$$
\begin{aligned}
\gamma & =\sqrt{\frac{m^{2}}{H^{2}}-\frac{9}{4}}, \\
\beta(m) & =-\frac{P_{i \gamma-1 / 2}^{1}\left(\cosh H z_{0}\right)}{Q_{i \gamma-1 / 2}^{1}\left(\cosh H z_{0}\right)},
\end{aligned}
$$

where $P_{\nu}^{\mu}$ and $Q_{\nu}^{\mu}$ are associated Legendre functions of the first kind and second kind respectively. We can determine the coefficient $C(m)$ by the normalization condition [28]

$$
2 \int_{z_{0}}^{\infty} d(H z) e^{-3 W} u_{m}(z) u_{m^{\prime}}^{*}(z)=\delta\left(\gamma^{\prime}-\gamma\right)
$$

as

$$
C(m)=\left(\frac{H}{\mu}\right)^{3 / 2} \frac{1}{\sqrt{\zeta(m)+\xi(m)}}
$$

where

$$
\zeta(m)=\left|\frac{\Gamma(i \gamma)}{\Gamma(i \gamma+1 / 2)}\right|^{2}, \xi(m)=\left|\frac{\Gamma(-i \gamma)}{\Gamma(-i \gamma+1 / 2)}+\pi \beta(m) \frac{\Gamma(i \gamma+1 / 2)}{\Gamma(i \gamma+1)}\right|^{2}
$$

The normalization of the time mode function is determined so that the correct canonical quantization of $\omega$ is ensured:

$$
v_{m}(\eta)=\frac{\sqrt{3 \kappa^{2}}}{-k^{2}} \frac{\sqrt{\pi}}{2}(-H \eta)^{-3 / 2} e^{-\gamma \pi / 2} H_{i \gamma}^{(1)}(-k \eta) .
$$

Next we calculate the vacuum expectation value for the energy density of the projected Weyl tensor $\delta E_{\mu \nu}$ generated by the KK modes with $m>3 H / 2$. The vacuum expectation value of $\delta \rho_{E}^{2}$ is given by

$$
\left\langle\left(\kappa_{4}^{2} \delta \rho_{E}(x)\right)^{2}\right\rangle=\frac{1}{(2 \pi)^{3}} \int d^{3} k \frac{k^{8}}{9} e^{-10 \alpha} \int_{0}^{\infty} d \gamma\left|u_{m}\left(z_{0}\right)\right|^{2}\left|v_{m}\right|^{2} .
$$

However we have to notice that in large $m$ and $k$ limit $\left|v_{m}\right|^{2}$ behaves

$$
\left|v_{m}\right|^{2} \sim \frac{3 \kappa^{2}}{k^{4}} \frac{(-H \eta)^{-3}}{2 \sqrt{(m / H)^{2}+(-k \eta)^{2}}} \quad(m, k \rightarrow \infty)
$$


This shows that $m$ integral in Eq.(55) has a logarithmic divergence. This ultraviolet divergence appears in the 5dimensional field theory even in Minkowski spacetime. Thus we have to subtract this divergence 28]. Then the correct vacuum expectation value becomes

$$
\left\langle\left(\kappa_{4}^{2} \delta \rho_{E}(x)\right)^{2}\right\rangle=\frac{1}{(2 \pi)^{3}} \int d^{3} k \frac{k^{8}}{9} e^{-10 \alpha} \int_{0}^{\infty} d \gamma\left|u_{m}\left(z_{0}\right)\right|^{2}\left(\left|v_{m}\right|^{2}-\frac{3 \kappa^{2}}{k^{4}} \frac{(-H \eta)^{-3}}{2 \sqrt{(m / H)^{2}+(-k \eta)^{2}}}\right)
$$

Then the power spectrum $P_{E}(k)$ is given by

$$
\begin{aligned}
P_{E}(k) & =\frac{1}{108 \pi^{2}}\left(\frac{k e^{-\alpha}}{H}\right)^{7}\left(\kappa_{4} H\right)^{2} C_{K K}^{2}, \\
C_{K K}^{2} & =\frac{H}{\mu}\left(1+\left(\frac{H}{\mu}\right)^{2}\right) \int_{0}^{\infty} d \gamma\left|u_{m}\left(z_{0}\right)\right|^{2}\left(\frac{\pi}{2} e^{-\gamma \pi}\left|H_{i \gamma}^{(1)}(-k \eta)\right|^{2}-\frac{1}{\sqrt{(m / H)^{2}+(-k \eta)^{2}}}\right) .
\end{aligned}
$$

The amplitude of $C_{K K}^{2}$ is enhanced for large $H / \mu$ as expected.

\section{B. Quantization of radion mode}

Next we discuss the quantization of the radion mode considered in [18]. The vacuum junction condition Eq. (44) is trivially satisfied for any $z$ by the bulk mode solution

$$
u_{\mathrm{r}} \propto e^{W}, m^{2}=m_{\mathrm{r}}^{2}=2 H^{2}
$$

This mode is non-normalizable in the single brane model. But in the two brane model, where the second brane is located at any fixed $z_{1}>z_{0}$, this mode is normalizable, and automatically satisfies the boundary condition at the second brane. This mode is identified as the radion in [18]. The time dependence of the radion mode is

$$
v_{\mathrm{r}}=(-k \eta)^{-3 / 2} B_{1 / 2}(-k \eta)
$$

Substituting the bulk mode function for the radion Eq. (60) into the second order action for $\Omega$ Eq.(34), we obtain the action for the time dependence of the radion as

$$
S_{\mathrm{r}}=\frac{N_{k}}{6 \kappa^{2}} \int d^{4} x e^{-3 \alpha}\left(\left(\Delta \dot{v}_{\mathrm{r}}\right)^{2}-e^{-2 \alpha} \Delta v_{\mathrm{r}} \Delta^{2} v_{\mathrm{r}}-H^{2}\left(\Delta v_{\mathrm{r}}\right)^{2}\right)
$$

where

$$
N_{k}=\int_{z_{0}}^{z_{1}} d z e^{-W}=\int_{z_{0}}^{z_{1}} d z \frac{\mu \sinh H z}{H}
$$

and we used the vacuum boundary condition for $\Omega, \mathcal{F}=0$. If we define a canonical 4-dimensional field

$$
\psi_{k}=\sqrt{\frac{N_{k}}{3 \kappa^{2}}} e^{-3 \alpha} \Delta v_{\mathrm{r}}
$$

this action becomes the form of a 4-dimensional scalar field with $m^{2}=2 H^{2}$ :

$$
S_{\mathrm{r}}=\frac{1}{2} \int d^{4} x e^{3 \alpha}\left(\dot{\psi}_{k}^{2}-e^{-2 \alpha} \psi_{k} \Delta \psi_{k}-H^{2} \psi_{k}^{2}\right) .
$$

The solution of $\psi_{k}$ is easily obtained including its normalization as

$$
\psi_{k}=\frac{\sqrt{\pi}}{2} H^{-1 / 2}(-H \eta)^{3 / 2} H_{1 / 2}^{(1)}(-k \eta)
$$

Using Eq. (66), the Weyl energy density perturbations for the quantum radion becomes

$$
\kappa_{4}^{2} \delta \rho_{E}=-\frac{\sqrt{\pi}}{2} \sqrt{\frac{\kappa^{2}}{3 N_{k}}} k^{2}(-H \eta)^{7 / 2} H^{-1 / 2} H_{1 / 2}^{(1)}(-k \eta) .
$$


This agrees with the result obtained by Gen and Sasaki 22].

A growing mode solution represents the tachyonic instability of de Sitter two branes. As is shown in Ref. [22], the tachyonic instability is not strong enough to cause gravitational instabilities on the brane in the sense that $P_{E}(k)$ does not grow. As noted in [18], a decaying mode also has an interesting physical meaning. The decaying mode corresponds to the dark radiation perturbation that is associated with a small black hole in the bulk [29]. After inflation, the dark radiaiton perturbation becomes a growing mode and this can affect CMB because the dark radiation perturbation induces isocurvature perturbations [30, 31]. The power spectrum $P_{E}(k)$ coming form the decaying mode determines the initial condition for this isocurvature perturbations. Taking the long wavelength limit $k e^{-\alpha} / H \rightarrow 0, P_{E}$ is given by

$$
\begin{aligned}
P_{E}(k) & =\frac{1}{108 \pi^{2}}\left(\frac{k e^{-\alpha}}{H}\right)^{8}\left(\kappa_{4} H\right)^{2} C_{\text {dark }}^{2}, \\
C_{\text {dark }}^{2} & =\left(\frac{H}{\mu}\right)^{2}\left(1+\left(\frac{H}{\mu}\right)^{2}\right) \frac{1}{\cosh H z_{1}-\cosh H z_{0}} .
\end{aligned}
$$

Although this could be large at high energies and/or when the second brane is close to the physical brane, the effect is negligible on large scales because the spectrum is highly blue tilted. However, in order to address observational consequences, a detailed analysis of the evolution of perturbations after inflation is needed.

\section{SCALAR FIELD ON THE BRANE}

In this section we include the scalar field perturbation $\delta \phi$ on the brane. We assume that the potential of the scalar field $\phi$ confined to the brane is very flat, so that the scalar field is slow-rolling. In such a situation, we can treat the corrections to the evolution of scalar field fluctuation due to the metric backreaction perturbatively. We first consider the problem of quantization of scalar perturbations using equations of motion based on the results of Ref. [18]. We show that the evolution equation for the Mukhanov-Sasaki variable has a correction term that comes from the bulk perturbations. To quantize the Mukhanov-Sasaki variable, we need its action coupled to the bulk gravitational field, which is derived in $\mathrm{Sec} \nabla \mathrm{B}$

\section{A. Equation of motion for scalar perturbations}

We expand the scalar field perturbations in terms of a slow-roll parameter;

$$
\delta \phi=\delta \phi_{0}+\delta \phi_{1}+\ldots
$$

The 0 -th order of the scalar field fluctuation obeys the following equation of motion,

$$
\delta \ddot{\phi}_{0}+3 H \delta \dot{\phi}_{0}+k^{2} e^{-2 \alpha} \delta \phi_{0}=0 .
$$

The metric perturbations are generated by the 0-th order fluctuation of the scalar field through the induced Einstein equations on the brane,

$$
\begin{aligned}
& 3 H \dot{\Psi}-3 H^{2} \Phi+k^{2} e^{-2 \alpha} \Psi=\frac{\kappa_{4, \mathrm{eff}}^{2}}{2}\left(\dot{\phi} \dot{\delta} \phi_{0}+V^{\prime} \delta \phi_{0}\right)+\frac{\kappa_{4}^{2}}{2} \delta \rho_{E}, \\
& H \Phi-\dot{\Psi}=\frac{\kappa_{4, \text { eff }}^{2}}{2} \dot{\phi} \delta \phi_{0}-\frac{\kappa_{4}^{2}}{2} \delta q_{E}, \\
& -\ddot{\Psi}-3 H \dot{\Psi}+H \dot{\Phi}+3 H^{2} \Phi-\frac{1}{3} k^{2} e^{-2 \alpha}(\Psi+\Phi)=\frac{\kappa_{4, \mathrm{eff}}^{2}}{2}\left(\dot{\phi} \dot{\delta} \dot{\phi}_{0}-V^{\prime} \delta \phi_{0}\right)+\frac{\kappa_{4}^{2}}{6} \delta \rho_{E}, \\
& -e^{-2 \alpha}(\Psi+\Phi)=\kappa_{4}^{2} \delta \pi_{E},
\end{aligned}
$$

where

$$
\begin{aligned}
\kappa_{4}^{2} \delta q_{E} & =\frac{k^{2} e^{-3 \alpha}}{3}(\dot{\Omega}-H \Omega), \\
\kappa_{4}^{2} \delta \pi_{E} & =\frac{e^{-3 \alpha}}{2}\left(\ddot{\Omega}-H \Omega+\frac{k^{2} e^{-2 \alpha}}{3} \Omega\right),
\end{aligned}
$$


and $\delta \rho_{E}$ is given by Eq. (45) These metric fluctuations in turn affect the dynamics of the 1st-order scalar field perturbation as

$$
\ddot{\delta} \phi_{1}+3 H \dot{\delta} \phi_{1}+k^{2} e^{-2 \alpha} \delta \phi_{1}=-V^{\prime \prime} \delta \phi_{0}-3 \dot{\phi} \dot{\Psi}+\dot{\phi} \dot{\Phi}-2 V^{\prime} \Phi .
$$

To calculate the quantum fluctuation of $\delta \phi_{1}$, we need to determine the metric perturbations, including the normalization. In the standard 4-dimensional cosmology, this can be fixed from the normalization of $\delta \phi_{0}$ by using the $(t, i)$ component of the 4-dimensional Einstein equations. However, in the brane-world, we can not determine $\Phi$ and $\Psi$ without the solution of $\Omega$ because there are the contributions from the projected Weyl tensor $\delta E_{\mu \nu}$.

Yet we can determine the normalization of a part of $\Phi$ and $\Psi$ only from $\delta \phi_{0}$. This can be shown as follows. First, we rewrite the expressions of $\Phi$ and $\Psi$, Eq.(20) and (23), using $\Omega$ and $\mathcal{F}$ as

$$
\begin{aligned}
& \Psi=\frac{e^{-\alpha-3 W}}{6}\left[3 W^{\prime} \mathcal{F}-3 H(\dot{\Omega}-H \Omega)-e^{-2 \alpha} \Delta \Omega\right], \\
& \Phi=\frac{e^{-\alpha-3 W}}{6}\left[-3 W^{\prime} \mathcal{F}-3 \ddot{\Omega}+6 H \dot{\Omega}-3 H^{2} \Omega+2 e^{-2 \alpha} \Delta \Omega\right] .
\end{aligned}
$$

Here we used the equation of motion for $\Omega$ to reduce the number of $z$-derivatives. Substituting these expressions into the induced Einstein equations (72)-(75), we obtain the equations written only by $\mathcal{F}$ and $\delta \phi_{0}$ :

$$
\begin{array}{r}
-3 H \dot{\mathcal{F}}-k^{2} e^{-2 \alpha} \mathcal{F}=\kappa^{2} e^{\alpha}\left(\dot{\phi} \delta \dot{\phi}_{0}+V^{\prime}(\phi) \delta \phi_{0}\right), \\
\dot{\mathcal{F}}=\kappa^{2} e^{\alpha} \dot{\phi} \delta \phi_{0}, \\
\ddot{\mathcal{F}}+2 H \dot{\mathcal{F}}=\kappa^{2} e^{\alpha}\left(\dot{\phi} \delta \dot{\phi}_{0}-V^{\prime}(\phi) \delta \phi_{0}\right) .
\end{array}
$$

These are the same as 4-dimensional Einstein equations if we define the quantities $\Phi_{4}$ and $\Psi_{4}$ by

$$
\mathcal{F}=-2 \frac{e^{\alpha}}{W^{\prime}} \Phi_{4}=2 \frac{e^{\alpha}}{W^{\prime}} \Psi_{4}
$$

We can interpret that $\mathcal{F}$ represents the 4-dimensional part of the metric variables and $\Omega$ contributes to them as a 5-dimensional correction. Using Eq. (82), we can fix the normalization of $\mathcal{F}$ from the quantization of $\delta \phi_{0}$ in the same manner as the 4-dimensional cosmology. However, this is not sufficient to determine the amplitude of metric perturbations $\Psi$ and $\Phi$. This is the limitation of the 4-dimensional effective theory. We must solve the bulk equation for $\Omega$.

The solution for $\Omega$ which satisfies the boundary condition on the brane is obtained in Ref. [18]. Combining the junction conditions, Eq. (81)-(83), we get an evolution equation for $\mathcal{F}$;

$$
\ddot{\mathcal{F}}-\left(H+2 \frac{\ddot{\phi}}{\dot{\phi}}\right) \dot{\mathcal{F}}+k^{2} e^{-2 \alpha} \mathcal{F}=0 .
$$

This gives the boundary condition for $\Omega$. The scalar field fluctuation $\delta \phi_{0}$ is written by $\mathcal{F}(t)$ as

$$
\kappa^{2} \delta \phi_{0}=e^{-\alpha} \frac{\dot{\mathcal{F}}}{\dot{\phi}} .
$$

The evolution equation for $\mathcal{F}$ Eq. (85) is consistent with the equation of motion for $\delta \phi_{0}$, Eq. (71). Assuming that $\phi$ is slow-rolling $|\ddot{\phi} / \dot{\phi}| \ll H$, the solution for $\mathcal{F}$ is

$$
\mathcal{F}(\eta)=C_{1} \frac{\cos (-k \eta)}{-k \eta}+C_{2} \frac{\sin (-k \eta)}{-k \eta} .
$$

This gives the boundary condition for $\Omega$. The solution for $\Omega$ in the bulk subject to this condition is given by

$$
\begin{aligned}
\Omega(z, \eta)= & C_{1} \sqrt{2 \pi} \sum_{l=0}^{\infty}(-1)^{l}\left(2 l+\frac{1}{2}\right) \frac{(\sinh H z)^{-1} Q_{2 l}(\cosh H z)}{\mu Q_{2 l}^{1}\left(\cosh H z_{0}\right)}(-k \eta)^{-3 / 2} J_{2 l+1 / 2}(-k \eta) \\
& +C_{2} \sqrt{2 \pi} \sum_{l=0}^{\infty}(-1)^{l}\left(2 l+\frac{3}{2}\right) \frac{(\sinh H z)^{-1} Q_{2 l+1}(\cosh H z)}{\mu Q_{2 l+1}^{1}\left(\cosh H z_{0}\right)}(-k \eta)^{-3 / 2} J_{2 l+3 / 2}(-k \eta) .
\end{aligned}
$$


Then it is possible to calculate the next order scalar field perturbations $\delta \phi_{1}$.

In order to evaluate the effect from metric perturbations, it is useful to use Mukhanov-Sasaki variable defined by

$$
Q=\delta \phi-\frac{\dot{\phi}}{H} \Psi
$$

In terms of slow-roll expansion, we have $Q_{0}=\delta \phi_{0}$ and $Q_{1}=\delta \phi_{1}-\frac{\dot{\phi}}{H} \Psi$. Then using the induced Einstein equations, Eq.(72), Eq.(74), and Eq.(75), we can derive the equation for $Q_{1}$;

$$
\ddot{Q}_{1}+3 H \dot{Q}_{1}+k^{2} e^{-2 \alpha} Q_{1}=-V^{\prime \prime} Q_{0}-6 \dot{H} Q_{0}+J
$$

where

$$
\begin{aligned}
J & =-\frac{\kappa_{4}^{2} \dot{\phi}}{3 H}\left(k^{2} \delta \pi_{E}+\delta \rho_{E}\right) \\
& =-\frac{\dot{\phi}}{H} \frac{k^{2} e^{-3 \alpha}}{6}\left(\ddot{\Omega}-H \dot{\Omega}+k^{2} e^{-2 \alpha} \Omega\right) .
\end{aligned}
$$

The equation is the same as the standard 4-dimensional cosmology except for the term $J$. $J$ describes the corrections that comes from the 5-dimensional bulk perturbations. In order to address the quantization of $Q$, we need the second order action for $Q$.

\section{B. Second order action for Mukhanov-Sasaki variable}

From now on, we derive the second order action for Mukhanov-Sasaki variable $Q$ coupled to the bulk metric perturbations described by the master variable $\Omega$. We first add the action of the 4-dimensional scalar field,

$$
S=\int d^{4} x \sqrt{-g_{4}}\left(-\frac{1}{2} \partial_{\mu} \phi \partial^{\mu} \phi-V(\phi)\right)
$$

to the gravitational part of the action. Perturbing the action Eq.92) up to second order, we get

$$
S=\frac{1}{2} \int d^{4} x e^{3 \alpha}\left[\delta \dot{\phi}^{2}+e^{-2 \alpha} \delta \phi \Delta \delta \phi-V^{\prime \prime} \delta \phi^{2}-2 \Phi\left(\dot{\phi} \delta \dot{\phi}+V^{\prime} \delta \phi\right)+6 \Psi\left(\dot{\phi} \delta \dot{\phi}-V^{\prime} \delta \phi\right)\right],
$$

where we took the longitudinal gauge and neglected the terms of second order in the metric perturbations since they are higher-order in the slow-roll parameter. Using Eq. (79) and (80), the total action becomes

$$
\begin{aligned}
S= & \int d^{5} x \frac{e^{-3 \alpha-3 W}}{6 \kappa^{2}}\left[(\Delta \dot{\Omega})^{2}-\left(\Delta \Omega^{\prime}\right)^{2}+e^{-2 \alpha} \Delta \Omega \Delta^{2} \Omega+\mu^{2} e^{2 W}(\Delta \Omega)^{2}\right] \\
& +\int d^{4} x \frac{e^{\alpha}}{6 \kappa^{2}}\left[\frac{9}{2} W^{\prime} \dot{\mathcal{F}}^{2}-\frac{3}{2} W^{\prime} e^{-2 \alpha} \mathcal{F} \Delta \mathcal{F}-W^{\prime} e^{-4 \alpha} \Omega \Delta^{2} \Omega\right] \\
& +\frac{1}{2} \int d^{4} x e^{3 \alpha}\left[\delta \dot{\phi}^{2}+e^{-2 \alpha} \delta \phi \Delta \delta \phi-V^{\prime \prime} \delta \phi^{2}+2 W^{\prime} \mathcal{F} e^{-\alpha}\left(2 \dot{\phi} \delta \dot{\phi}-V^{\prime} \delta \phi\right)\right. \\
& \left.+\dot{\phi} \delta \dot{\phi} e^{-\alpha}\left(\ddot{\Omega}-5 H \dot{\Omega}+4 H^{2} \Omega-\frac{5}{3} e^{-2 \alpha} \Delta \Omega\right)+V^{\prime} \delta \phi e^{-\alpha}\left(\ddot{\Omega}+H \Omega-2 H^{2} \Omega+\frac{1}{3} e^{-2 \alpha} \Delta \Omega\right)\right]
\end{aligned}
$$

Taking the variation of this second order action with respect to $\Omega$, we get the equation of motion for $\Omega$ in the bulk and the junction condition:

$$
e^{-2 \alpha} \Delta \mathcal{F}=\kappa^{2} e^{\alpha} \dot{\phi} \delta \dot{\phi}
$$

where we used the equation of motion for scalar field at the zeroth order in the slow-roll parameter. From Eq.95), we can derive Eq.(82). We also get another junction condition by minimizing the action with respect to $\mathcal{F}$ as

$$
3(\ddot{\mathcal{F}}+\dot{\alpha} \dot{\mathcal{F}})+e^{-2 \alpha} \Delta \mathcal{F}=\kappa^{2} e^{\alpha}\left(4 \dot{\phi} \delta \dot{\phi}-2 V^{\prime} \delta \phi\right) .
$$

Combining the junction conditions, we get an evolution equation for $\mathcal{F}$, Eq. (85). 
Now we derive the action for $Q$. It is useful to notice that the terms contain $\mathcal{F}$ in Eq. (94) are the same as the 4-dimensional theory if we rewrite $\mathcal{F}$ by $\Psi_{4}$ using Eq. (84). Thus it is convenient to define $Q_{4}$ as

$$
Q_{4}=\delta \phi-\frac{\dot{\phi}}{H} \Psi_{4}=\delta \phi-\frac{\dot{\phi}}{H} \frac{W^{\prime}}{2} e^{-\alpha} \mathcal{F}
$$

Using Eq.95) and (97) to express $\dot{\mathcal{F}}, \delta \phi$ and $\delta \dot{\phi}$ in terms of $\mathcal{F}, Q_{4}$ and $\dot{Q}_{4}$, we obtain the following action:

$$
\begin{aligned}
S= & \int d^{5} x \frac{e^{-3 \alpha-3 W}}{6 \kappa^{2}}\left[(\Delta \dot{\Omega})^{2}-\left(\Delta \Omega^{\prime}\right)^{2}+e^{-2 \alpha} \Delta \Omega \Delta^{2} \Omega+\mu^{2} e^{2 W}(\Delta \Omega)^{2}\right] \\
& +\frac{1}{2} \int d^{4} x e^{3 \alpha}\left[\dot{Q}_{4}{ }^{2}+e^{-2 \alpha} Q_{4} \Delta Q_{4}-\left(V^{\prime \prime}+6 \dot{H}\right) Q_{4}^{2}\right. \\
& \left.-\frac{W^{\prime}}{3 \kappa^{2}} e^{-6 \alpha}(\Delta \Omega)^{2}-\dot{\phi} e^{-\alpha}\left(\dddot{\Omega}-3 H^{2} \dot{\Omega}+2 H^{3} \Omega-e^{-2 \alpha} \Delta\left(\frac{5}{3} \dot{\Omega}-H \Omega\right)\right) Q_{4}\right] .
\end{aligned}
$$

If we express $\mathcal{F}$ in Eq.(97) by $\Psi$ and $\Omega$ using Eq.(79), we immediately get the action in terms of $Q$ and $\dot{Q}$ as

$$
\begin{aligned}
S= & \int d^{5} x \frac{e^{-3 \alpha-3 W}}{6 \kappa^{2}}\left[(\Delta \dot{\Omega})^{2}-\left(\Delta \Omega^{\prime}\right)^{2}+e^{-2 \alpha} \Delta \Omega \Delta^{2} \Omega+\mu^{2} e^{2 W}(\Delta \Omega)^{2}\right] \\
& +\frac{1}{2} \int d^{4} x e^{3 \alpha}\left[\dot{Q}^{2}+e^{-2 \alpha} Q \Delta Q-\left(V^{\prime \prime}+6 \dot{H}\right) Q^{2}\right. \\
& \left.-\frac{W^{\prime} e^{-6 \alpha}}{3 \kappa^{2}}(\Delta \Omega)^{2}+\frac{\dot{\phi} e^{-3 \alpha}}{3 H} Q \Delta\left(\ddot{\Omega}-H \dot{\Omega}-e^{-2 \alpha} \Delta \Omega\right)\right] .
\end{aligned}
$$

We shoud note that the slow-roll approximation was used to derive Eq.(98) and (99). The equation of motion for $Q$ can be easily derived from this action:

$$
\ddot{Q}+3 H \dot{Q}-e^{-2 \alpha} \Delta Q=-\left(V^{\prime \prime}+6 \dot{H}\right) Q+\frac{\dot{\phi} e^{-3 \alpha}}{6 H} \Delta\left(\ddot{\Omega}-H \dot{\Omega}-e^{-2 \alpha} \Delta \Omega\right) .
$$

Of course, this agrees with Eq.(90). We can also derive the junction condition for $\Omega$ :

$$
e^{-2 \alpha} \Delta \mathcal{F}=\kappa^{2} e^{\alpha} \dot{\phi} \dot{Q}
$$

The action (99) is the main result of this paper. This action describes the coupling between the matter fields on the brane and gravitational fields in the bulk. Although the 5-dimensional gravitational fields are very complicated, the final action is very simple if we use the master variable. Essentially the system is described by two scalar fields. One is living on the brane and the other 5-dimensional scalar field is living in the bulk and they are coupled with each other on the brane.

In general it is very difficult to quantize this coupled system of the bulk and the brane. However, from the solution for $\Omega(88)$, we can see that the coupling term is suppressed by the slow-roll parameter. Thus it is possible to solve the equations perturbatively. At the 0 -th order, the scalar field perturbation $Q_{0}=\delta \phi_{0}$ decouples. Then by quantizing $Q_{0}$, we can determine the coefficient $C_{1}$ and $C_{2}$ in Eq. (87) as

$$
C_{1}=\kappa^{2} \frac{i \dot{\phi}}{\sqrt{2 k} H}, C_{2}=i C_{1}
$$

where we chose a standard Bunch-Davis vacuum. The master variable $\Omega$ couples to $Q_{0}$ via the junction condition Eq.(101). Then the normalization of $\Omega$ is also determined from the solutions Eq. (88). There are also contributions from the normalizable KK modes with $m>3 H / 2$ which satisfy the vacuum junction condition $\mathcal{F}=0$. The contribution of the KK continuum with $m>3 H / 2$ to the bulk perturbations are the same as the case of the vacuum brane, so the normalization is determined as in SecIVA With these KK modes as well as the discrete modes induced by the scalar field on the brane, we can calculate the correction term $J$, which is needed to know the behavior of $Q_{1}$.

Because there is a contribution from the infinite ladder of modes in $J$, the contribution of $J$ could be significant especially at high energies $H / \mu \gg 1$ on small scales. In this case we should carefully reexamine our perturbation scheme. A quantification of the 5-dimensional corrections is beyond the scope of our present paper and we hope to report results in future publications. 


\section{SUMMARY AND DISCUSSION}

In this paper, we considered the quantum scalar perturbation about a de Sitter brane in a 5-dimensional AdS bulk spacetime. We first introduced the gauge-invariant master variable $\Omega$ for gravitational perturbations in the AdS spacetime found by Mukohyama 19]. We then derived its second order action which is needed to perform the quantization of the master variable.

In the case of a vacuum single de Sitter brane, there is a continuum of normalizable KK modes with $m>3 H / 2$. These bulk perturbations are felt on the brane through the projection of the perturbed 5-dimensional Weyl tensor $\delta E_{\mu \nu}$. We calculated the vacuum expectation value of its effective energy density by using the second order action for $\Omega$. A light radion mode with $m=\sqrt{2} H$ also satisfies the junction conditions on the branes and is normalizable for a two branes model. The energy density of $\delta E_{\mu \nu}$ due to this radion mode is also calculated and is shown to agree with the previous results obtained by Gen and Sasaki [22].

Next we considered the case where there is a scalar field perturbation on a single de Sitter brane. The bulk perturbations can be solved using the slow-roll approximation [18]. As shown in [18], the $m^{2}=2 H^{2}$ mode together with the zero-mode and an infinite ladder of discrete tachyonic modes become normalizable. There are also contributions from the continuum of the KK modes which satisfy the vacuum boundary condition on the brane $\mathcal{F}=0$. These bulk perturbations introduce corrections to the scalar type perturbations of the standard 4-dimensional cosmology, as is seen from Eq.(90). To calculate the amplitude of scalar perturbations on the brane, we need to compute the vacuum expectation value of Mukhanov-Sasaki variable $Q$. Then we derived the sencond order action (99) for $Q$ coupled to 5-dimensional bulk perturbations. Because the coupling term between $Q$ and the master variable $\Omega$ is suppressed by a slow-roll parameter, we can solve the system by a slow-roll expansion.

The action (99) describes the essential features of scalar perturbations in the brane world. The scalar field perturbation on the brane inevitably produces the bulk gravitational perturbations, which back-react to the perturbations on the brane. A detailed analysis of this coupled bulk-brane system is very important in order to find brane wolrd signatures from inflation and we hope to come back to this issue in future publications.

\section{Acknowledgments}

H.Y. acknowledges valuable discussions with Shinji Mukohyama. The works of H.Y. and K.K. are supported by Grant-in-Aid for JSPS fellows.

[1] K. i. Maeda and M. Sasaki, (eds.), Brane world:New perspective in cosmology, Prog.Theor. Phys. Supplement, 148 (2002).

[2] D. Langlois, Prog. Theor. Phys. Suppl. 148, 181 (2003).

[3] R. Maartens, Liv. Rev. Rel. 7, 1 (2004).

[4] P. Brax, C. van de Bruck and A. C. Davis, arXiv:hep-th/0404011

[5] L. Randall and R. Sundrum, Phys. Rev. Lett. 83, 3370 (1999).

[6] L. Randall and R. Sundrum, Phys. Rev. Lett. 83, 4690 (1999).

[7] K. Koyama and J. Soda, Phys. Rev. D 62 (2000) 123502; K. Koyama and J. Soda, Phys. Rev. D 65 (2002) 023514.

[8] K. Koyama, JCAP, to appear arXiv:astro-ph/0407263.

[9] N. Kaloper, Phys. Rev. D 60, 123506 (1999).

[10] J. Garriga and M. Sasaki, Phys. Rev. D 62, 043523 (2000).

[11] H. A. Bridgman, K. A. Malik, and D. Wands, Phys. Rev. D 63, 084012 (2001).

[12] D. Langlois, R. Maartens, and D. Wands, Phys. Lett. B 489, 259 (2000).

[13] D. S. Gorbunov, V. A. Rubakov and S. M. Sibiryakov, JHEP 0110, 015 (2001).

[14] A. Frolov and L. Kofman, arXiv:hep-th/0209133

[15] T. Kobayashi, H. Kudoh and T. Tanaka, Phys. Rev. D 68, 044025 (2003).

[16] R. Maartens, D. Wands, B. A. Bassett, and I. Heard, Phys. Rev. D 62, 041301 (2000).

[17] E. Ramirez and A. R. Liddle, Phys. Rev. D 69, 083522 (2004)

[18] K. Koyama, D. Langlois, R. Maartens, and D. Wands, hep-th/0408222

[19] S. Mukohyama, Phys. Rev. D 62, 084015 (2000).

[20] H. Kodama, A. Ishibashi and O. Seto, Phys. Rev. D 62, 064022 (2000).

[21] U. Gen and M. Sasaki, Prog. Theor. Phys. 105, 591 (2001)

[22] U. Gen and M. Sasaki, Prog. Theor. Phys. 108, 471 (2002).

[23] P. Binetruy, C. Deffayet and D. Langlois, Nucl. Phys. B 565, 269 (2000).

[24] T. Shiromizu, K. i. Maeda and M. Sasaki, Phys. Rev. D 62, 024012 (2000).

[25] H. A. Bridgman, K. A. Malik and D. Wands, Phys. Rev. D 65, 043502 (2002). 
[26] C. van de Bruck, M. Dorca, R. H. Brandenberger and A. Lukas, Phys. Rev. D 62, 123515 (2000).

[27] C. Deffayet, Phys. Rev. D 66, 103504 (2002).

[28] S. Kobayashi, K. Koyama and J. Soda, Phys. Lett. B 501, 157 (2001)

[29] H. Yoshiguchi and K. Koyama, Phys. Rev. D, 70, 043513.

[30] D. Langlois, R. Maartens, M. Sasaki and D. Wands, Phys. Rev. D 63, 084009 (2001)

[31] K. Koyama, Phys. Rev. Lett. 91, 221301 (2003). 